\title{
ON THE EXTREMAL THEORY OF CONTINUED FRACTIONS
}

\author{
ALINA BAZAROVA ${ }^{1}$, ISTVÁN BERKES ${ }^{2}$, AND LAJOS HORVÁTH ${ }^{3}$
}

\begin{abstract}
Letting $x=\left[a_{1}(x), a_{2}(x), \ldots\right]$ denote the continued fraction expansion of an irrational number $x \in(0,1)$, Khinchin proved that $S_{n}(x)=\sum_{k=1}^{n} a_{k}(x) \sim \frac{1}{\log 2} n \log n$ in measure, but not for almost every $x$. Diamond and Vaaler showed that removing the largest term from $S_{n}(x)$, the previous asymptotics will hold almost everywhere, showing the crucial influence of the extreme terms of $S_{n}(x)$ on the sum. In this paper we determine, for $d_{n} \rightarrow \infty, d_{n} / n \rightarrow 0$, the precise asymptotics of the sum of the $d_{n}$ largest terms of $S_{n}(x)$ and show that the sum of the remaining terms has an asymptotically Gaussian distribution.
\end{abstract}

\section{Introduction}

For an irrational number $x \in(0,1)$ let

$$
x=\frac{1}{a_{1}+\frac{1}{a_{2}+\frac{1}{\ddots}}}
$$

be the continued fraction expansion of $x$. Clearly

$$
a_{1}(x)=[1 / x], \quad a_{n+1}(x)=a_{1}\left(T^{n} x\right), \quad n \geq 1,
$$

where the transformation $T:(0,1) \rightarrow(0,1)$ is defined by $T x=\{1 / x\}$; here $[\cdot]$ and $\{\cdot\}$ denote integral resp. fractional part. Let

$$
\mu(E)=\frac{1}{\log 2} \int_{E} \frac{1}{1+x} d x
$$

be the Gauss measure on the class $\mathcal{B}$ of Borel subsets of $(0,1)$. It is known (see e.g. [3]) that $T$ is an ergodic transformation preserving the Gauss measure and thus with respect to the probability

\footnotetext{
2010 Mathematics Subject Classification. Primary 11K50, 60F05, 60G70.

Key words and phrases. Continued fraction expansion, extreme elements, mixing random variables, central limit theorem.

${ }^{1}$ Graz University of Technology, Institute of Statistics, Kopernikusgasse 24, 8010 Graz, Austria. e-mail: bazarova@tugraz.at. Research supported by Austrian Science Fund (FWF) Grant W1230.

${ }^{2}$ Graz University of Technology, Institute of Statistics, Kopernikusgasse 24, 8010 Graz, Austria. e-mail: berkes@tugraz .at. Research supported by Austrian Science Fund (FWF) Grant P24302-N18.

${ }^{3}$ University of Utah, Department of Mathematics, Salt Lake City, UT 84112-0090, USA. e-mail: horvath@math.utah.edu. Research supported by NSF grant DMS-13-05858.
} 
space $((0,1), \mathcal{B}, \mu),\left\{a_{n}(x), n \geq 1\right\}$ is a stationary ergodic sequence. Clearly, the set $\left\{a_{1}=k\right\}$ is the interval $(1 /(k+1), 1 / k]$ and thus

$$
\mu\left\{a_{1}=k\right\}=\frac{1}{\log 2} \int_{1 /(k+1)}^{1 / k} \frac{1}{1+x} d x=\frac{1}{\log 2} \log \left\{1+\frac{1}{k(k+2)}\right\} \sim \frac{1}{\log 2} \frac{1}{k^{2}} .
$$

(We say that $a_{k} \sim b_{k}$ if $\lim _{k \rightarrow \infty} a_{k} / b_{k}=1$.) Thus by the ergodic theorem we have for any function $F: \mathbb{N} \rightarrow \mathbb{R}$

$$
\lim _{N \rightarrow \infty} \frac{1}{N} \sum_{k=1}^{N} F\left(a_{k}(x)\right)=\frac{1}{\log 2} \sum_{j=1}^{\infty} F(j) \log \left\{1+\frac{1}{j(j+2)}\right\} \quad \text { a.e. }
$$

provided that the series on the right hand side converges absolutely.

The sequence $\left\{a_{k}(x), k \geq 1\right\}$ has remarkable mixing properties. Gauss noted that the distribution of $T^{n} x=\left[a_{n+1}(x), a_{n+2}(x), \ldots\right]$ with respect to the uniform measure in $(0,1)$ converges to $\mu$ and asked for the speed of convergence. (For a discussion, see [3], pp. 49-50 or [17], p. 552.) Kusmin [19] showed that the convergence speed is $O\left(e^{-\lambda \sqrt{k}}\right)$ and Lévy [21] improved this to $O\left(e^{-\lambda k}\right)$. Lévy's result implies that the sequence $\left\{a_{k}(x), k \geq 1\right\}$ is $\psi$-mixing with exponential rate, i.e. for all $A \in \mathcal{F}_{1}^{k}$, $B \in \mathcal{F}_{k+n}^{\infty}, k \geq 1, n \geq 1$ we have

$$
|\mu(A \cap B)-\mu(A) \mu(B)| \leq C e^{-\lambda n} \mu(A) \mu(B)
$$

with positive absolute constants $C, \lambda$, where $\mathcal{F}_{r}^{s}$ denotes the $\sigma$-field generated by the variables $\left\{a_{k}(x), r \leq k \leq s\right\}$.

Letting $\mathrm{E}$ denote expectation with respect to $\mu$, we have $\mathrm{E} a_{1}=\infty$ and correspondingly for $F(x)=x$ the right hand side of (1.1) is $+\infty$. Thus the partial sums $\sum_{k=1}^{N} a_{k}(x)$ grow faster than $N$. Lévy [22] proved that

$$
\frac{1}{N} \sum_{k=1}^{N} a_{k}(x)-\frac{\log N}{\log 2} \stackrel{d}{\longrightarrow} G,
$$

where $\stackrel{d}{\longrightarrow}$ means convergence in distribution in the probability space $((0,1), \mathcal{B}, \mu)$, and $G$ is a stable distribution with characteristic function

$$
\exp \left(-\frac{\pi|t|}{2 \log 2}-\frac{i t \log |t|}{\log 2}-\frac{i \kappa t}{\log 2}\right)
$$

where $\kappa=0.577 \ldots$ is the Euler-Mascheroni constant. See also Theorem 2, pp. 159-160 of Heinrich [14], where a remainder term estimate for the convergence in (1.2) is obtained. This implies that

$$
\lim _{N \rightarrow \infty} \frac{1}{N \log N} \sum_{k=1}^{N} a_{k}(x)=\frac{1}{\log 2} \quad \text { in measure, }
$$

a result obtained earlier by Khinchin [18]. Khinchin also noted that (1.4) cannot hold almost everywhere. Diamond and Vaaler [9] showed that the obstacle to a.e. convergence in (1.4) is the occurrence of one single large term in the sum $\sum_{k=1}^{N} a_{k}(x)$ and established an a.e. analogue of (1.4) by excluding the largest summand. They proved namely

$$
\lim _{N \rightarrow \infty} \frac{1}{N \log N} S_{N}^{(1)}(x)=\frac{1}{\log 2} \quad \text { for almost all } x
$$


where $S_{N}^{(d)}(x)$ denotes the sum $\sum_{k=1}^{N} a_{k}(x)$ after discarding its $d$ largest summands. The proof shows that (1.5) remains valid if $S_{N}^{(1)}$ is replaced by $S_{N}^{(d)}$ for any fixed $d \geq 2$ and discarding more terms improves the rate of a.e. convergence in (1.5). An analogous result for the St. Petersburg game was proved by Csörgö and Simons [7]. For further analogies between continued fraction digits and the St. Petersburg game we refer to Vardi [32]. In view of these facts it is natural to ask what happens if from the sum $S_{N}=\sum_{k=1}^{N} a_{k}(x)$ we remove $d=d_{N}$ terms, where

$$
d_{N} \rightarrow \infty, \quad d_{N} / N \rightarrow 0
$$

so that the number of discarded terms is 'large', but is still negligible compared with $N$. The purpose of this paper is to answer this question. Let

$$
m(t)=\frac{1}{\log 2} \sum_{1 \leq k \leq t} k \log \left(1+\frac{1}{k(k+2)}\right), \quad t \geq 1 .
$$

We will prove the following result.

Theorem 1.1. Let $d=d_{N}$ satisfy (1.6). Then we have

$$
\frac{S_{N}^{(d)}-N m\left(\eta_{d, N}\right)}{N / \sqrt{d}} \stackrel{d}{\longrightarrow} N\left(0,(\log 2)^{-1}\right)
$$

where $\eta_{d, N}$ denotes the $d$-th largest of $a_{1}, \ldots, a_{N}$ and $N\left(\mu, \sigma^{2}\right)$ denotes the normal distribution with mean $\mu$ and variance $\sigma^{2}$.

Theorem 1.1 reduces the asymptotic study of $S_{N}^{(d)}$ to that of $\eta_{d, N}$, which is a much simpler problem. We will show in (3.15) that $\eta_{d, N} \sim N / d$ in probability and since $m(t) \sim(\log 2)^{-1} \log t$ as $t \rightarrow \infty$, Theorem 1.1 can be rewritten equivalently as

$$
S_{N}^{(d)}=N m\left(\eta_{d, N}\right)+(N / \sqrt{d}) \zeta_{N}=\left(1+o_{P}(1)\right) \frac{1}{\log 2} N \log (N / d)+(N / \sqrt{d}) \zeta_{N},
$$

where $\zeta_{N} \stackrel{d}{\longrightarrow} N(0,1 / \log 2)$. Here and in the sequel, $\stackrel{P}{\longrightarrow}$ will denote convergence in probability and $o_{P}(1)$ a quantity converging to 0 in probability. Relation (1.9) shows that $N m\left(\eta_{d, N}\right)$ is the main term in an asymptotic expansion of $S_{N}^{(d)}$. As a comparison, write Lévy's limit theorem (1.2) in the form

$$
S_{N}=\frac{1}{\log 2} N \log N+N \zeta_{N}^{*},
$$

where $\zeta_{N}^{*}$ converges in distribution to the Cauchy variable with characteristic function (1.3). In addition to the change of the order of magnitude of $S_{N}$ caused by removing the $d$ largest terms, note that the Cauchy fluctuations of $S_{N}$ around $\frac{1}{\log 2} N \log N$ described by (1.10) changed to Gaussian fluctuations around $N m\left(\eta_{d, N}\right)$ in (1.9). An immediate consequence of relation (1.9) is

$$
\frac{S_{N}^{(d)}}{N \log (N / d)} \stackrel{P}{\longrightarrow} \frac{1}{\log 2}
$$

under (1.6). If $d$ grows slower than any power of $N$, i.e. $\log d / \log N \rightarrow 0$, then the last relation implies

$$
\frac{1}{N \log N} S_{N}^{(d)} \stackrel{P}{\longrightarrow} \frac{1}{\log 2} .
$$


Thus in this case the order of magnitude of $S_{N}^{(d)}$ is the same as that of the complete sum $S_{N}$, i.e. the contribution of the $d$ largest terms of $S_{N}$ is still negligible compared to the whole sum. If $d \sim N^{\gamma}$ for some $0<\gamma<1$, then

$$
\frac{1}{N \log N} S_{N}^{(d)} \stackrel{P}{\rightarrow} \frac{1-\gamma}{\log 2}
$$

We thus see that the removal of of a small portion of extreme elements of $S_{N}$ changes the asymptotic order of magnitude of the sum, hence the role of large elements in $S_{N}$ is very substantial.

In case of i.i.d. variables in the domain of attraction of a stable law with parameter $0<\alpha<2$, the effect of the extremal terms on the partial sums is well known. For positive variables Darling [8] showed (see also Arov and Bobrov [1]) that under some additional regularity assumptions the ratio of the sum and its largest term has a non-degenerate limit distribution if $0<\alpha<1$ and this holds also for $1<\alpha<2$ provided we center the partial sum by its mean. The case $\alpha=1$ is critical and is not covered in [1], [8]. The sequence $\left\{a_{k}(x), k \geq 1\right\}$ in the continued fraction expansion corresponds to this case, except that the variables $a_{k}$ are weakly dependent. Theorem 1.1 and its corollaries above show that the contribution of the $d$ largest terms of $S_{N}$ is negligible (in probability) compared with the total sum $S_{N}$ if and only if $\log d / \log N \rightarrow 0$. In particular this holds for $d=1$, i.e. in the case of the largest term. In the i.i.d. case, Csörgö, Horváth and Mason [6] also showed that removing the $d$ largest and $d$ smallest elements from the partial sum, where (1.6) holds, the remaining sum $S_{N}^{(d)}$ becomes asymptotically normal. Our Theorem 1.1 is a dependent analogue of this result for continued fractions. There is a large literature on the metric properties of continued fractions and using the exponential $\psi$-mixing property of the transformation $T$ above, many classical limit theorems for partial sums of independent random variables have been extended to continued fractions. We refer to Doeblin [10], Gordin and Reznik [13], Ibragimov [15], Iosifescu [16], [17], Philipp [23], [25], Philipp and Stackelberg [26], Samur [27], [28], Stackelberg [29], Szewczak [30] and the references therein. Using the extremal theory of dependent processes, (see e.g. Leadbetter and Rootzen [20]), asymptotic properties of the (individual) extremes of $\left(a_{1}(x), \ldots, a_{n}(x)\right)$ can be established; limit theorems for the largest digit were obtained by Galambos [11], [12], Philipp [24]. Note that an analogue of Theorem 1.1 for a different, less natural trimming of the partial quotients $a_{j}$ was obtained in Philipp [25].

In Section 2, we will prove Theorem 1.1 in a probabilistic form and we will change the notation accordingly.

Theorem 1.2. Let $\left\{X_{j}, j \geq 1\right\}$ be a strictly stationary sequence of positive, integer valued random variables with

$$
P\left(X_{1}=k\right) \sim c_{0} k^{-2} \quad \text { as } \quad k \rightarrow \infty
$$

for some constant $c_{0}>0$. Assume that $\left\{X_{j}, j \geq 1\right\}$ is $\psi$-mixing with rate $\psi(n)=C e^{-\lambda n}$ for some $C>0, \lambda>0$. Let $\eta_{d, n}$ denote the $d$-th largest of $X_{1}, \ldots, X_{n}$ and assume that $d=d_{n}$ satisfies (1.6). Let $m(t)=\mathrm{E} X_{1} I\left\{X_{1} \leq t\right\}$ and

$$
A_{n}=\sqrt{c_{0}} n / \sqrt{d}
$$

Then

$$
\frac{1}{A_{n}} \sum_{i=1}^{[n t]}\left(X_{i} I\left\{X_{i} \leq \eta_{d, n}\right\}-m\left(\eta_{d, n}\right)\right) \stackrel{\mathcal{D}[0,1]}{\longrightarrow} W(t),
$$


where $W$ is the Wiener process.

Remark 1.1. If $\left(X_{n}\right)$ is a sequence of positive random variables such that with probability one $X_{1}, X_{2}, \ldots$ are different, then the sum $\sum_{i=1}^{[n t]} X_{i} I\left\{X_{i} \leq \eta_{d, n}\right\}$ in (1.13) is obtained from $\sum_{i=1}^{[n t]} X_{i}$ by removing the $d-1$ largest terms and thus the conclusion of Theorem 1.2 for $t=1$ reduces to that of Theorem 1.1. However, for integer valued variables $X_{n}, \eta_{d, n}$ can appear in the sequence $\left(X_{1}, \ldots, X_{n}\right)$ more than once and in this case the number of terms of the sum $\sum_{i=1}^{[n t]} X_{i}$ exceeding $\eta_{n, d}$ can be smaller than $d-1$ and can actually be random. Thus, in a formal sense, Theorem 1.1 is not a special case of Theorem 1.2. However, using a simple perturbation argument Theorem 1.1 will be deduced from Theorem 1.2.

Let

$$
U_{n}(t, s)=\sum_{i=1}^{[n t]}\left(X_{i} I\left\{X_{i} \leq s(n / d)\right\}-E X_{i} I\left\{X_{i} \leq s(n / d)\right\}\right) \quad(t \geq 0, s \geq 0) .
$$

We will derive Theorem 1.2 from the following two-dimensional limit theorem.

Theorem 1.3. Under the assumptions of Theorem 1.2 we have

$$
\frac{1}{A_{n}} U_{n}(t, s) \longrightarrow W(t, s) \text { weakly in } \mathcal{D}([0,1] \times[1 / 2,3 / 2]),
$$

where $\{W(t, s), t \geq 0, s \geq 0\}$ is a two-parameter Wiener process.

As we already noted, under (1.6) we have

$$
\frac{\eta_{d, n}}{n / d} \longrightarrow 1 \quad \text { in probability. }
$$

Since the limit process $W(t, s)$ in (1.14) has continuous trajectories a.s., Theorem 1.3 and Billingsley [4], p. 144-145 imply that

$$
\frac{1}{A_{n}} U_{n}\left(t, \eta_{d, n} /(n / d)\right) \stackrel{\mathcal{D}[0,1]}{\longrightarrow} W(t, 1)
$$

which is exactly the functional central limit theorem (1.13).

In conclusion we note that Theorem 1.2 and Theorem 1.3 remain valid assuming a suitable polynomial $\psi$-mixing rate instead of the exponential rate. However, as this requires extensive changes in the arguments and we do not know of any practically interesting examples for $\psi$-mixing sequences with polynomial rate, we omit the details.

\section{Some lemmas}

In the rest of the paper $\left(X_{k}\right)$ denotes a sequence of random variables satisfying the conditions of Theorem 1.2 and $d=d_{n}$ denotes a sequence of positive integers satisfying (1.6). Moreover, $c_{0}$ denotes the constant in (1.11). Given a process $Y(s, t)$ defined on a rectangle $H=[a, b] \times\left[a^{\prime}, b^{\prime}\right]$, let $Y(H)$ denote the increment of $Y$ over $H$. 
Lemma 2.1. Let $\left\{Y_{n}(t, s), n \geq 1\right\}$ be processes defined on a rectangle $[a, b] \times\left[a^{\prime}, b^{\prime}\right] \subset[0, \infty)^{2}$ and assume that for some $\gamma>0$

$$
\mathrm{E}\left|Y_{n}(B)\right|^{\gamma}\left|Y_{n}(C)\right|^{\gamma} \leq \mu(B) \mu(C),
$$

where $\mu$ denotes area and $B$ and $C$ are rectangles of the form $\left[t_{1}, t_{2}\right] \times\left[s_{1}, s_{2}\right]$ having one common edge, but otherwise disjoint. Then the sequence $\left\{Y_{n}(t, s), n \geq 1\right\}$ is tight. If every $X_{n}(t, s)$ is piecewise constant in $t$, i.e. there exists a finite set $H_{n} \subset[a, b]$ such that $X_{n}(t, s)$ is constant in the left closed intervals determined by the elements of $H_{n} \cup\{a\} \cup\{b\}$, then it suffices to verify (2.1) for rectangles $\left[t_{1}, t_{2}\right] \times\left[s_{1}, s_{2}\right]$ where $t_{1}, t_{2} \in H_{n}$.

This is a special case of a general tightness condition in Bickel and Wichura [2].

Lemma 2.2. Let $X, Y$ be integrable random variables such that $X$ is measurable with respect to $\sigma\left(X_{1}, \ldots, X_{k}\right)$ and $Y$ is measurable with respect to $\sigma\left(X_{k+n}, X_{k+n+1}, \ldots\right)$. Then $X Y$ is also integrable and

$$
|\mathrm{E} X Y-\mathrm{E} X \mathrm{E} Y| \leq \psi(n) \mathrm{E}|X| \mathrm{E}|Y|
$$

This follows from Theorem 3.10 in Bradley [5], p. 75.

Lemma 2.3. Let $\mathcal{G}_{k}$ denote the $\sigma$-field generated by $X_{k}$, let $n_{1}<\ldots<n_{r}$ be positive integers and let $Y_{1}, \ldots, Y_{r}$ be bounded r.v.'s such that $Y_{j}$ is $\mathcal{G}_{n_{j}}$ measurable $(j=1,2, \ldots, r)$. Then

$$
E\left|Y_{1} \cdots Y_{r}\right| \leq C_{r} E\left|Y_{1}\right| \cdots E\left|Y_{r}\right|,
$$

where $C_{r}=(1+\psi(1))^{r}$.

Proof. This is immediate by induction upon observing that by the previous lemma we have for any $1 \leq j \leq r-1$

$$
\mathrm{E}\left|Y_{1} \cdots Y_{j+1}\right| \leq \mathrm{E}\left|Y_{1} \cdots Y_{j}\right| \mathrm{E}\left|Y_{j+1}\right|+\psi(1) \mathrm{E}\left|Y_{1} \cdots Y_{j}\right| \mathrm{E}\left|Y_{j+1}\right|=(1+\psi(1)) \mathrm{E}\left|Y_{1} \cdots Y_{j}\right| \mathrm{E}\left|Y_{j+1}\right| .
$$

Lemma 2.4. For any $T \geq 3$ we have

$$
\mathrm{E} X_{1} I\left\{X_{1} \leq T\right\} \leq C_{1} \log T, \quad \mathrm{E} X_{1}^{4} I\left\{X_{1} \leq T\right\} \leq C_{1} T^{3} .
$$

Moreover, for any fixed $0 \leq s_{1}<s_{2}$ we have

$$
\mathrm{E} X_{1}^{2} I\left\{s_{1}(n / d)<X_{1} \leq s_{2}(n / d)\right\} \sim c_{0}\left(s_{2}-s_{1}\right)(n / d) \quad \text { as } n \rightarrow \infty
$$

and for any fixed $0<s_{1}<s_{2}$ and sufficiently large $n$

$$
\mathrm{E} X_{1} I\left\{s_{1}(n / d)<X_{1} \leq s_{2}(n / d)\right\} \leq C_{2}\left(s_{2}-s_{1}\right) / s_{1} .
$$

Here $C_{1}, C_{2}$ are positive constants depending only on the sequence $\left(X_{k}\right)$.

This is immediate from (1.11).

Lemma 2.5. Let

$$
X_{k, n}^{\left(s_{1}, s_{2}\right)}=X_{k} I\left\{s_{1}(n / d)<X_{k} \leq s_{2}(n / d)\right\}-\mathrm{E} X_{k} I\left\{s_{1}(n / d)<X_{k} \leq s_{2}(n / d)\right\} .
$$


Then for any fixed $0 \leq t_{1}<t_{2} \leq 1,0 \leq s_{1}<s_{2}<\infty$ we have

$$
\mathrm{E}\left(\sum_{k=n t_{1}+1}^{n t_{2}} X_{k, n}^{\left(s_{1}, s_{2}\right)}\right)^{2} \sim c_{0}\left(n^{2} / d\right)\left(t_{2}-t_{1}\right)\left(s_{2}-s_{1}\right) \quad \text { as } \quad n \rightarrow \infty
$$

provided $n t_{1}, n t_{2}$ are integers. Moreover,

$$
\mathrm{E}\left(\sum_{i=n t_{1}+1}^{n t_{2}} X_{i, n}^{\left(s_{1}, s_{2}\right)}\right)\left(\sum_{j=n t_{1}^{\prime}+1}^{n t_{2}^{\prime}} X_{j, n}^{\left(s_{1}^{\prime}, s_{2}^{\prime}\right)}\right)=o\left(n^{2} / d\right) \quad \text { as } \quad n \rightarrow \infty
$$

provided $0 \leq t_{1}<t_{2} \leq 1,0 \leq t_{1}^{\prime}<t_{2}^{\prime} \leq 1,0 \leq s_{1}<s_{2}<\infty, 0 \leq s_{1}^{\prime}<s_{2}^{\prime}<\infty, n t_{1}, n t_{2}, n t_{1}^{\prime}, n t_{2}^{\prime}$ are integers and the intervals $\left(n t_{1}, n t_{2}\right)$ and $\left(n t_{1}^{\prime}, n t_{2}^{\prime}\right)$ are identical or disjoint and the same holds for the intervals $\left(s_{1}, s_{2}\right)$ and $\left(s_{1}^{\prime}, s_{2}^{\prime}\right)$, but identity cannot hold at both places.

Proof. We have

$$
\mathrm{E}\left(\sum_{k=n t_{1}+1}^{n t_{2}} X_{k, n}^{\left(s_{1}, s_{2}\right)}\right)^{2}=n\left(t_{2}-t_{1}\right) \mathrm{E}\left(X_{1, n}^{\left(s_{1}, s_{2}\right)}\right)^{2}+R
$$

where

$$
R=2 \sum_{j=2}^{n t_{2}-n t_{1}}\left(n t_{2}-n t_{1}-j+1\right) \mathrm{E}\left(X_{1, n}^{\left(s_{1}, s_{2}\right)} X_{j, n}^{\left(s_{1}, s_{2}\right)}\right) .
$$

Using Lemmas 2.2 and 2.4 we get, using $n / d \rightarrow \infty$,

$$
\begin{aligned}
& \mathrm{E}\left(X_{1, n}^{\left(s_{1}, s_{2}\right)}\right)^{2}=\mathrm{E}\left(X_{1} I\left\{s_{1}(n / d)<X_{1} \leq s_{2}(n / d)\right\}\right)^{2}-\mathrm{E}^{2}\left(X_{1} I\left\{s_{1}(n / d)<X_{1} \leq s_{2}(n / d)\right\}\right) \\
& =c_{0}(1+o(1))(n / d)\left(s_{2}-s_{1}\right)+O\left(\log ^{2}(n / d)\right) \sim c_{0}(n / d)\left(s_{2}-s_{1}\right)
\end{aligned}
$$

and

$$
|R| \leq 2 n \sum_{j=2}^{n t_{2}-n t_{1}} \psi(j-1)\left(\mathrm{E}\left|X_{1, n}^{\left(s_{1}, s_{2}\right)}\right|\right)^{2} \leq C_{3} n \log ^{2}(n / d) \sum_{j=2}^{\infty} e^{-\lambda j}=o\left(n^{2} / d\right),
$$

proving (2.5).

To prove $(2.6)$, consider a generic term

$$
\begin{aligned}
& \mathrm{E} X_{i, n}^{\left(s_{1}, s_{2}\right)} X_{j, n}^{\left(s_{1}^{\prime}, s_{2}^{\prime}\right)} \\
& \quad=\mathrm{E} X_{i} X_{j} I\left\{s_{1}(n / d)<X_{i} \leq s_{2}(n / d)\right\} I\left\{s_{1}^{\prime}(n / d)<X_{j} \leq s_{2}^{\prime}(n / d)\right\} \\
& \quad-\mathrm{E} X_{i} I\left\{s_{1}(n / d)<X_{i} \leq s_{2}(n / d)\right\} \mathrm{E} X_{j} I\left\{s_{1}^{\prime}(n / d)<X_{j} \leq s_{2}^{\prime}(n / d)\right\}
\end{aligned}
$$

of the left hand side of (2.6). Fix $r \geq 0$ and sum those covariances in (2.7) where $j-i=r$ and $n t_{1}+1 \leq i \leq n t_{2}, n t_{1}^{\prime}+1 \leq j \leq n t_{2}^{\prime}$. Clearly, the case $r=0$ can occur only if $\left(n t_{1}, n t_{2}\right)=\left(n t_{1}^{\prime}, n t_{2}^{\prime}\right)$, but in this case by the assumptions of the lemma $\left(s_{1}, s_{2}\right)$ and $\left(s_{1}^{\prime}, s_{2}^{\prime}\right)$ must be disjoint and thus the product of the two indicators in the second line of (2.7) is 0 . Thus by the first statement of Lemma 2.4 the product expectation in the first line of $(2.7)$ is $O\left(\log ^{2}(n / d)\right)$ and since the number of such terms in the expansion of (2.6) is at most $n$, the contribution of such terms in the sum in (2.6) is at most $O\left(n \log ^{2}(n / d)\right)=o\left(n^{2} / d\right)$ by $n / d \rightarrow \infty$. For $r \geq 1$ the covariance in (2.7) is at 
most $\psi(r) O\left(\log ^{2}(n / d)\right)$ by Lemma 2.2 and the first statement of Lemma 2.4 and since for fixed $r$ the number of pairs $(i, j)$ is at most $n$, the contribution of all such terms for all $r \geq 1$ is at most $C n \log ^{2}(n / d) \sum_{r=1}^{\infty} \psi(r)=O\left(n \log ^{2}(n / d)\right)=o\left(n^{2} / d\right)$, proving $(2.6)$.

The following central limit theorem for $\phi$-mixing sequences is due to Utev [31].

Lemma 2.6. Let $\left\{x_{n k}, 1 \leq k \leq n, n \geq 1\right\}$ be a triangular array of random variables with zero mean and finite variances. Assume that the array is $\phi$-mixing, i.e.

$$
\phi(k):=\sup _{n \in \mathbb{N}, n>k} \max _{1 \leq m \leq n-k} \sup _{A \in \mathcal{F}_{1, m}^{(n)}, B \in \mathcal{F}_{m+k, n}^{(n)}, P(A)>0}|P(B \mid A)-P(B)| \longrightarrow 0 \quad \text { as } k \rightarrow \infty
$$

where $\mathcal{F}_{a, b}^{(n)}$ denotes the $\sigma$-algebra generated by the r.v.'s $\left\{x_{n k}, a \leq k \leq b\right\}$. Assume further that

$$
\lim _{n \rightarrow \infty} \mathrm{E}\left(\sum_{k=1}^{n} x_{n k}\right)^{2}=\sigma^{2}<\infty
$$

and that the Lindeberg condition

$$
\lim _{n \rightarrow \infty} n \mathrm{E}\left[x_{n 1}^{2} I\left(\left|x_{n 1}\right| \geq \varepsilon\right)\right]=0 \quad \text { for all } \varepsilon>0
$$

holds. Then

$$
\sum_{k=1}^{n} x_{n k} \stackrel{d}{\longrightarrow} N\left(0, \sigma^{2}\right) \quad \text { as } n \rightarrow \infty
$$

\section{Proof of Theorem 1.3}

Put

$$
Q_{n}=\frac{1}{A_{n}} \sum_{m=1}^{M} \sum_{j=1}^{J} \mu_{m, j} U_{n}\left(\left[t_{m-1}, t_{m}\right] \times\left[s_{j-1}, s_{j}\right]\right)
$$

and

$$
Z=\sum_{m=1}^{M} \sum_{j=1}^{J} \mu_{m, j} W\left(\left[t_{m-1}, t_{m}\right] \times\left[s_{j-1}, s_{j}\right]\right)
$$

for all $M \geq 1, J \geq 1$, real coefficients $\mu_{m, j}, 0=s_{0}<s_{1}<s_{2}<\ldots<s_{J}<\infty, 0=t_{0}<t_{1}<\ldots<$ $t_{M}=1$. Clearly, $Z$ is a normal random variable with mean zero and

$$
\mathrm{E} Z^{2}=\sum_{m=1}^{M} \sum_{j=1}^{J} \mu_{m, j}^{2}\left(t_{m}-t_{m-1}\right)\left(s_{j}-s_{j-1}\right) .
$$

We claim that

$$
Q_{n} \stackrel{d}{\longrightarrow} Z \quad \text { for all considered values of } M, J, \mu_{m, j}, t_{m}, s_{j} .
$$

Since the processes $U_{n}$ and $W$ are equal to 0 on the boundary of the first quadrant, we have

$$
U_{n}\left(t_{m}, s_{j}\right)=\sum_{p=1}^{m} \sum_{q=1}^{j} U_{n}\left(\left[t_{p-1}, t_{p}\right] \times\left[s_{q-1}, s_{q}\right]\right)
$$


and the same relation holds for $W$. Thus (3.2) implies

$$
\frac{1}{A_{n}} \sum_{m=1}^{M} \sum_{j=1}^{J} \mu_{m, j}^{*} U_{n}\left(t_{m}, s_{j}\right) \stackrel{d}{\longrightarrow} \sum_{m=1}^{M} \sum_{j=1}^{J} \mu_{m, j}^{*} W\left(t_{m}, s_{j}\right)
$$

for arbitrary real coefficients $\mu_{m, j}^{*}$ and this, by the Cramér-Wold device, implies the convergence of the finite-dimensional distributions in Theorem 1.3.

Clearly, $U_{n}\left(\left[t_{m-1}, t_{m}\right] \times\left[s_{j-1}, s_{j}\right]\right)$ equals

$$
\sum_{k=\left[n t_{m-1}\right]+1}^{\left[n t_{m}\right]} X_{k} I\left\{s_{j-1}(n / d)<X_{k} \leq s_{j}(n / d)\right\}-\mathrm{E} X_{k} I\left\{s_{j-1}(n / d)<X_{k} \leq s_{j}(n / d)\right\}
$$

and thus relation (3.2) is equivalent to

$$
\frac{1}{A_{n}} \sum_{k=1}^{n}\left(z_{n k}-E z_{n k}\right) \stackrel{d}{\longrightarrow} N\left(0, E Z^{2}\right)
$$

where

$$
z_{n k}=\sum_{j=1}^{J} \mu_{m, j} X_{k} I\left\{s_{j-1}(n / d)<X_{k} \leq s_{j}(n / d)\right\}, \quad\left[n t_{m-1}\right]+1 \leq k \leq\left[n t_{m}\right] .
$$

Since the terms of the sum in (3.4) are random variables with disjoint support, by relation (2.3) of Lemma 2.4 we have

$$
\mathrm{E} z_{n k}^{2}=\left(1+o_{n}(1)\right) c_{0}(n / d) \sum_{j=1}^{J} \mu_{m, j}^{2}\left(s_{j}-s_{j-1}\right), \quad\left[n t_{m-1}\right]+1 \leq k \leq\left[n t_{m}\right] .
$$

Consequently, letting

$$
B_{m}=c_{0} \sum_{j=1}^{J} \mu_{m, j}^{2}\left(s_{j}-s_{j-1}\right)
$$

we get

$$
\operatorname{Var} z_{n k} \leq\left(1+o_{n}(1)\right)(n / d) B_{m}, \quad\left[n t_{m-1}\right]+1 \leq k \leq\left[n t_{m}\right] .
$$

Further, Lemma 2.5 implies for $n \rightarrow \infty$

$$
\mathrm{E} U_{n}\left(\left[t_{m-1}, t_{m}\right] \times\left[s_{j-1}, s_{j}\right]\right)^{2}=\left(1+o_{n}(1)\right) c_{0}\left(t_{m}-t_{m-1}\right)\left(s_{j}-s_{j-1}\right)\left(n^{2} / d\right)
$$

and

$$
\mathrm{E} U_{n}\left(\left[t_{m_{1}-1}, t_{m_{1}}\right] \times\left[s_{j_{1}-1}, s_{j_{1}}\right]\right) U_{n}\left(\left[t_{m_{2}-1}, t_{m_{2}}\right] \times\left[s_{j_{2}-1}, s_{j_{2}}\right]\right)=o_{n}\left(n^{2} / d\right)
$$

provided the pairs $\left(m_{1}, j_{1}\right)$ and $\left(m_{2}, j_{2}\right)$ are different. Thus

$$
\begin{aligned}
& \mathrm{E}\left(\sum_{k=1}^{n}\left(z_{n k}-\mathrm{E} z_{n k}\right)\right)^{2}=\mathrm{E}\left(\sum_{m=1}^{M} \sum_{j=1}^{J} \mu_{m, j} U_{n}\left(\left[t_{m-1}, t_{m}\right] \times\left[s_{j-1}, s_{j}\right]\right)\right)^{2} \\
(3.7)= & \sum_{m_{1}, m_{2}=1}^{M} \sum_{j_{1}, j_{2}=1}^{J} \mu_{m_{1}, j_{1}} \mu_{m_{2}, j_{2}} \mathrm{E}\left[U_{n}\left(\left[t_{m_{1}-1}, t_{m_{1}}\right] \times\left[s_{j_{1}-1}, s_{j_{1}}\right]\right) U_{n}\left(\left[t_{m_{2}-1}, t_{m_{2}}\right] \times\left[s_{j_{2}-1}, s_{j_{2}}\right]\right)\right]
\end{aligned}
$$




$$
\sim c_{0}\left(n^{2} / d\right) \sum_{m=1}^{M} \sum_{j=1}^{J} \mu_{m, j}^{2}\left(t_{m}-t_{m-1}\right)\left(s_{j}-s_{j-1}\right)=c_{0}\left(n^{2} / d\right) \mathrm{E} Z^{2}=A_{n}^{2} \mathrm{E} Z^{2} .
$$

Also, by (3.4) and the second relation of (2.2) we have

$$
\left\|z_{n k}\right\|_{4} \leq \sum_{j=1}^{J}\left|\mu_{m, j}\right|\left(\mathrm{E} X_{k}^{4} I\left\{s_{j-1}(n / d)<X_{k} \leq s_{j}(n / d)\right\}\right)^{1 / 4} \leq C_{1}^{1 / 4}(n / d)^{3 / 4} \sum_{j=1}^{J}\left|\mu_{m, j}\right| s_{j}^{3 / 4}
$$

and consequently

$$
\left\|z_{n k}-\mathrm{E} z_{n k}\right\|_{4} \leq 2 C_{1}^{1 / 4}(n / d)^{3 / 4} \sum_{j=1}^{J}\left|\mu_{m, j}\right| s_{j}^{3 / 4} .
$$

We apply now Lemma 2.6 for the triangular array

$$
x_{n k}=\left(z_{n k}-\mathrm{E} z_{n k}\right) / A_{n}, \quad 1 \leq k \leq n, n \geq 1 .
$$

Since $\left\{X_{j}, j \geq 1\right\}$ is $\psi$-mixing with exponential rate, the array (3.9) satisfies the $\phi$-mixing condition (2.8). Relation (3.7) shows that (2.9) holds with $\sigma^{2}=\mathrm{E} Z^{2}$. Finally, (3.8), (1.12) and $d=d_{n} \rightarrow \infty$ show that the array (3.9) satisfies the Ljapunov condition

$$
\lim _{n \rightarrow \infty} n \mathrm{E} x_{n 1}^{4}=0
$$

which implies the Lindeberg condition (2.10). Thus Lemma 2.6 applies and the central limit theorem (3.3) follows.

Next we prove tightness in Theorem 1.3. Let

$$
B_{11}=\left[t_{1}, t\right] \times\left[s_{1}, s\right], \quad B_{12}=\left[t_{1}, t\right] \times\left[s, s_{2}\right], \quad B_{21}=\left[t, t_{2}\right] \times\left[s_{1}, s\right] .
$$

where $0 \leq t_{1}<t<t_{2} \leq 1,1 / 2 \leq s_{1}<s<s_{2} \leq 3 / 2$. In view of Lemma 2.1, it suffices to show that

$$
\mathrm{E}\left|\frac{1}{A_{n}} U_{n}\left(B_{11}\right)\right|^{2}\left|\frac{1}{A_{n}} U_{n}\left(B_{i j}\right)\right|^{2} \leq C^{*} \mu\left(B_{11}\right) \mu\left(B_{i j}\right)
$$

holds for each $i j \in\{12,21\}$ with some constant $C^{*}>0$. Moreover, since $U_{n}(t, s)$ is constant on intervals $k / n \leq t<(k+1) / n$, by the last statement of Lemma 2.1 we may assume that $n t, n t_{1}$ and $n t_{2}$ are all integers. To prove (3.11), we introduce the notations

$$
\begin{array}{ll}
X_{i}^{(1)}=X_{i} I\left\{s_{1}(n / d)<X_{i} \leq s(n / d)\right\}, & m_{i}^{(1)}=\mathrm{E} X_{i}^{(1)}, \\
X_{i}^{(2)}=X_{i} I\left\{s(n / d)<X_{i} \leq s_{2}(n / d)\right\}, & m_{i}^{(2)}=\mathrm{E} X_{i}^{(2)} .
\end{array}
$$

Using Lemmas 2.3 and 2.5 and (1.12) we get

$$
\begin{aligned}
& \mathrm{E}\left|\frac{1}{A_{n}} U_{n}\left(B_{11}\right)\right|^{2}\left|\frac{1}{A_{n}} U_{n}\left(B_{21}\right)\right|^{2} \\
& =\mathrm{E}\left(\frac{1}{A_{n}} \sum_{i=n t_{1}+1}^{n t}\left(X_{i}^{(1)}-m_{i}^{(1)}\right)\right)^{2}\left(\frac{1}{A_{n}} \sum_{i=n t+1}^{n t_{2}}\left(X_{i}^{(1)}-m_{i}^{(1)}\right)\right)^{2} \\
& \leq(1+\psi(1))^{2} \frac{1}{A_{n}^{4}} \mathrm{E}\left(\sum_{i=n t_{1}+1}^{n t}\left(X_{i}^{(1)}-m_{i}^{(1)}\right)\right)^{2} \mathrm{E}\left(\sum_{i=n t+1}^{n t_{2}}\left(X_{i}^{(1)}-m_{i}^{(1)}\right)\right)^{2}
\end{aligned}
$$




$$
\leq C_{8}\left(t-t_{1}\right)\left(t_{2}-t\right)\left(s-s_{1}\right)^{2}=C_{8} \mu\left(B_{11}\right) \mu\left(B_{21}\right) .
$$

for $n \geq n_{0}$. On the other hand,

$$
\begin{aligned}
& \mathrm{E}\left|\frac{1}{A_{n}} U_{n}\left(B_{11}\right)\right|^{2}\left|\frac{1}{A_{n}} U_{n}\left(B_{12}\right)\right|^{2} \\
& =\frac{1}{A_{n}^{4}} \mathrm{E}\left(\sum_{i=n t_{1}+1}^{n t}\left(X_{i}^{(1)}-m_{i}^{(1)}\right)\right)^{2}\left(\sum_{i=n t_{1}+1}^{n t}\left(X_{i}^{(2)}-m_{i}^{(2)}\right)\right)^{2} \\
& =\frac{1}{A_{n}^{4}} \mathrm{E}\left(\sum_{i=n t_{1}+1}^{n t} Y_{i}^{(1)}\right)^{2}\left(\sum_{i=n t_{1}+1}^{n t} Y_{i}^{(2)}\right)^{2}
\end{aligned}
$$

where we put

$$
Y_{i}^{(1)}=X_{i}^{(1)}-m_{i}^{(1)}, \quad Y_{i}^{(2)}=X_{i}^{(2)}-m_{i}^{(2)}
$$

The expression in the third line of (3.12) equals the sum of all expressions

$$
A_{n}^{-4} \mathrm{E}\left(Y_{i}^{(1)} Y_{j}^{(1)} Y_{k}^{(2)} Y_{\ell}^{(2)}\right)
$$

where $n t_{1}+1 \leq i, j, k, \ell \leq n t$. The following facts can be verified by elementary calculations using Lemmas 2.2-2.4:

(a) $\mathrm{E}\left|Y_{i}^{(1)}\right| \ll s-s_{1}, \quad \mathrm{E}\left|Y_{i}^{(2)}\right| \ll s_{2}-s, \quad \mathrm{E}\left|Y_{i}^{(1)} Y_{i}^{(2)}\right| \ll\left(s-s_{1}\right)\left(s_{2}-s\right)$

(b) $\mathrm{E}\left(Y_{i}^{(1)}\right)^{2} \ll(n / d)\left(s-s_{1}\right), \quad \mathrm{E}\left(Y_{i}^{(2)}\right)^{2} \ll(n / d)\left(s_{2}-s\right)$,

(c) $\mathrm{E}\left(Y_{i}^{(1)}\right)^{2}\left|Y_{i}^{(2)}\right| \ll(n / d)\left(s-s_{1}\right)\left(s_{2}-s\right), \quad \mathrm{E}\left|Y_{i}^{(1)}\right|\left(Y_{i}^{(2)}\right)^{2} \ll(n / d)\left(s-s_{1}\right)\left(s_{2}-s\right)$,

(d) $\mathrm{E}\left(Y_{i}^{(1)}\right)^{2}\left(Y_{i}^{(2)}\right)^{2} \ll(n / d)\left(s-s_{1}\right)\left(s_{2}-s\right)$,

where $\ll$ means the same as the $O$ notation, with an implied constant depending on the sequence $\left(X_{n}\right)$. We prove relation (d), the proof of (a), (b), (c) is similar (and simpler). We have

$$
\begin{aligned}
& \mathrm{E}\left(Y_{i}^{(1)}\right)^{2}\left(Y_{i}^{(2)}\right)^{2}=\mathrm{E}\left[\left(X_{i}^{(1)}-m_{i}^{(1)}\right)^{2}\left(X_{i}^{(2)}-m_{i}^{(2)}\right)^{2}\right] \\
& =\mathrm{E}\left(X_{i}^{(1)}\right)^{2}\left(X_{i}^{(2)}\right)^{2}-2 m_{i}^{(2)} \mathrm{E}\left(X_{i}^{(1)}\right)^{2} X_{i}^{(2)}+\left(m_{i}^{(2)}\right)^{2} \mathrm{E}\left(X_{i}^{(1)}\right)^{2}-2 m_{i}^{(1)} \mathrm{E} X_{i}^{(1)}\left(X_{i}^{(2)}\right)^{2} \\
& +4 m_{i}^{(1)} m_{i}^{(2)} \mathrm{E} X_{i}^{(1)} X_{i}^{(2)}-2 m_{i}^{(1)}\left(m_{i}^{(2)}\right)^{2} \mathrm{E} X_{i}^{(1)}+\left(m_{i}^{(1)}\right)^{2} \mathrm{E}\left(X_{i}^{(2)}\right)^{2}-2\left(m_{i}^{(1)}\right)^{2} m_{i}^{(2)} \mathrm{E} X_{i}^{(2)} \\
& +\left(m_{i}^{(1)}\right)^{2}\left(m_{i}^{(2)}\right)^{2} .
\end{aligned}
$$

Clearly $X_{i}^{(1)}$ and $X_{i}^{(2)}$ are supported on different sets and thus $X_{i}^{(1)} X_{i}^{(2)}=0$. Thus among the 9 terms above, the first, second, fourth and fifth are equal to 0 . Also, the second and third statement of Lemma 2.4 imply, in view of $1 / 2 \leq s_{1}<s<s_{2} \leq 3 / 2$,

$$
\begin{aligned}
& m_{i}^{(1)}=\mathrm{E} X_{1}^{(1)} \ll s-s_{1}, \quad m_{i}^{(2)}=\mathrm{E} X_{i}^{(2)} \ll s_{2}-s \\
& \mathrm{E}\left(X_{i}^{(1)}\right)^{2} \ll\left(s-s_{1}\right)(n / d), \quad \mathrm{E}\left(X_{i}^{(2)}\right)^{2} \ll\left(s_{2}-s\right)(n / d)
\end{aligned}
$$

for $n \geq n_{0}$. This shows that the remaining five terms of the sum above are $\ll(n / d)\left(s-s_{1}\right)\left(s_{2}-s\right)$, proving statement (d) above. Statements (a), (b) and (c) can be proved similarly.

We can now estimate the expressions in (3.13). We will distinguish four cases according as $i, j, k, \ell$ are all different, or the number of different ones among them is 1,2 or 3 . Consider first 
the case when $i, j, k, l$ are all different, say $i<j<k<\ell$; let $r=j-i$. Applying Lemma 2.2 with $X=Y_{i}^{(1)}, Y=Y_{j}^{(1)} Y_{k}^{(2)} Y_{\ell}^{(2)}$ and using that $E X=0$, we get that the absolute value of the expression (3.13) is bounded by

$$
A_{n}^{-4} \psi(r) \mathrm{E}|X| \mathrm{E}|Y| \leq C A_{n}^{-4} \psi(r) \mathrm{E}\left|Y_{i}^{(1)}\right| \mathrm{E}\left|Y_{j}^{(1)}\right| \mathrm{E}\left|Y_{k}^{(2)}\right| \mathrm{E}\left|Y_{\ell}^{(2)}\right| \leq C A_{n}^{-4} \psi(r)\left(s-s_{1}\right)^{2}\left(s_{2}-s\right)^{2},
$$

where we used Lemma 2.3 to estimate $\mathrm{E}|Y|$ and relation (a) above. Here, and in the rest of the tightness proof, $C$ denotes (possibly different) constants depending only on the sequence $\left(X_{n}\right)$. Arguing similarly, but splitting the four-term product in (3.13) after the third term, we get the same bound, except that $\psi(r)$ gets replaced by $\psi\left(r^{\prime}\right)$, where $r^{\prime}=\ell-k$. Thus the absolute value of the expression in (3.13) is at most

$$
C A_{n}^{-4} \psi(r)^{1 / 2} \psi\left(r^{\prime}\right)^{1 / 2}\left(s-s_{1}\right)^{2}\left(s_{2}-s\right)^{2} .
$$

Fixing the pair $(i, \ell)$ and summing for $(j, k)$ means summing for $\left(r, r^{\prime}\right)$ and since $\sum_{n=1}^{\infty} \psi(n)^{1 / 2}<\infty$ and the pair $(i, \ell)$ can be chosen by at most $\left(n t-n t_{1}\right)^{2}$ different ways, it follows that the contribution of all terms (3.13) with $i<j<k<\ell$ is at most

$$
\begin{aligned}
& C A_{n}^{-4}\left(n t-n t_{1}\right)^{2}\left(s-s_{1}\right)^{2}\left(s_{2}-s\right)^{2} \leq C\left(d^{2} / n^{2}\right)\left(t-t_{1}\right)^{2}\left(s-s_{1}\right)^{2}\left(s_{2}-s\right)^{2} \\
& \leq C\left(t-t_{1}\right)^{2}\left(s-s_{1}\right)\left(s_{2}-s\right)=C \mu\left(B_{11}\right) \mu\left(B_{12}\right),
\end{aligned}
$$

using (1.12) and $d / n \rightarrow 0$. The contribution of terms (3.13) where $i, j, k, \ell$ are different, but their order is different can be estimated similarly.

Next we consider the case when $i=j=k=\ell$. In this case the expression (3.13) becomes $A_{n}^{-4} E\left(Y_{i}^{(1)}\right)^{2} E\left(Y_{i}^{(2)}\right)^{2}$, which by the estimate in (d) above is at most $C A_{n}^{-4}(n / d)\left(s-s_{1}\right)\left(s_{2}-s\right)$. Since the number of choices for $i$ is $n t-n t_{1} \leq\left(n t-n t_{1}\right)^{2}$, the contribution of all such expressions is bounded by

$$
C A_{n}^{-4}(n / d)\left(s-s_{1}\right)\left(s_{2}-s\right)\left(n t-n t_{1}\right)^{2} \leq C(d / n)\left(s-s_{1}\right)\left(s_{2}-s\right)\left(t-t_{1}\right)^{2} \leq C \mu\left(B_{11}\right) \mu\left(B_{12}\right),
$$

using again (1.12) and $d / n \rightarrow 0$.

Assume now that among $i, j, k, \ell$ there are two different ones, i.e. these numbers are pairwise equal or three are equal and the fourth is different. Starting with the case of two pairs, assume e.g. that $i=j$ and $k=l$, but $i \neq k$. In this case the expression (3.13) becomes $A_{n}^{-4} \mathrm{E}\left(Y_{i}^{(1)}\right)^{2}\left(Y_{k}^{(2)}\right)^{2}$ which, in view of Lemma 2.3 and the estimate in (b) above is at most

$$
C A_{n}^{-4}(n / d)^{2}\left(s-s_{1}\right)\left(s_{2}-s\right) \text {. }
$$

Since the number of choices for the pair $(i, k)$ is at most $\left(n t-n t_{1}\right)^{2}$, using (1.12) it follows that the total contribution of all such terms (3.13) is at most

$$
C A_{n}^{-4}(n / d)^{2}\left(s-s_{1}\right)\left(s_{2}-s\right)\left(n t-n t_{1}\right)^{2} \leq C\left(s-s_{1}\right)\left(s_{2}-s\right)\left(t-t_{1}\right)^{2}=C \mu\left(B_{11}\right) \mu\left(B_{12}\right) .
$$

If $i=k, j=l$ and $i \neq j$, then the expression (3.13) becomes $A_{n}^{-4} \mathrm{E} Y_{i}^{(1)} Y_{i}^{(2)} Y_{j}^{(1)} Y_{j}^{(2)}$ which by Lemma 2.3 and the estimate in (a) above is bounded by

$$
C A_{n}^{-4} \mathrm{E}\left|Y_{i}^{(1)} Y_{i}^{(2)}\right| \mathrm{E}\left|Y_{j}^{(1)} Y_{j}^{(2)}\right| \leq C A_{n}^{-4}\left(s-s_{1}\right)^{2}\left(s_{2}-s\right)^{2} .
$$

Since the number of pairs $(i, j)$ is $\leq\left(n t-n t_{1}\right)^{2}$, the contribution of such terms is at most

$$
C A_{n}^{-4}\left(s-s_{1}\right)^{2}\left(s_{2}-s\right)^{2}\left(n t-n t_{1}\right)^{2} \leq C\left(s-s_{1}\right)\left(s_{2}-s\right)\left(t-t_{1}\right)^{2}=C \mu\left(B_{11}\right) \mu\left(B_{12}\right) .
$$


Assume now that from the indices $i, j, k, l$ three are equal and the fourth one is different. Letting e.g. $i=j=k$ and $i \neq \ell$, the expression (3.13) becomes $A_{n}^{-4} \mathrm{E}\left(Y_{i}^{(1)}\right)^{2} Y_{i}^{(2)} Y_{\ell}^{(2)}$ which is, by Lemma 2.3 and the estimates (a) and (c) above is bounded by

$$
C A_{n}^{-4} \mathrm{E}\left(Y_{i}^{(1)}\right)^{2}\left|Y_{i}^{(2)}\right| \mathrm{E}\left|Y_{\ell}^{(2)}\right| \leq C A_{n}^{-4}(n / d)\left(s-s_{1}\right)\left(s_{2}-s\right)^{2} .
$$

Since the number of pairs $(i, \ell)$ is $\leq\left(n t-n t_{1}\right)^{2}$, the total contribution of such terms is at most $C \mu\left(B_{11}\right) \mu\left(B_{12}\right)$.

Finally, if the number of different indices among $i, j, k, l$ is 3 , e.g. if $i=j<k<\ell$, then the expression (3.13) becomes $A_{n}^{-4} \mathrm{E}\left(Y_{i}^{(1)}\right)^{2} Y_{k}^{(2)} Y_{\ell}^{(2)}$ which by using $\mathrm{E} Y_{\ell}^{(2)}=0$, Lemma 2.2, Lemma 2.3 and estimates (a) and (b) above, can be estimated by

$$
C A_{n}^{-4} \psi(r) \mathrm{E}\left(Y_{i}^{(1)}\right)^{2} \mathrm{E}\left|Y_{k}^{(2)}\right| \mathrm{E}\left|Y_{\ell}^{(2)}\right| \leq C A_{n}^{-4} \psi(r)(n / d)\left(s-s_{1}\right)\left(s_{2}-s\right)^{2},
$$

where $r=\ell-k$. Since for fixed $r$ the number of triples $(i, k, \ell)$ with $\ell-k=r$ is at most $\left(n t-n t_{1}\right)^{2}$, the contribution of such terms (3.13) is at most

$$
C A_{n}^{-4} \psi(r)(n / d)\left(s-s_{1}\right)\left(s_{2}-s\right)^{2}\left(n t-n t_{1}\right)^{2} \leq C \psi(r)\left(s-s_{1}\right)\left(s_{2}-s\right)\left(t-t_{1}\right)^{2}
$$

and summing for $r$ we get again $\leq C \mu\left(B_{11}\right) \mu\left(B_{12}\right)$. The other cases (e.g. $i<j=k<\ell$, etc.) can be treated similarly and the proof of tightness in Theorem 1.3 is completed. This also completes the proof of the theorem.

We prove now, as claimed after Theorem 1.3, that

$$
\frac{\eta_{d, n}}{n / d} \stackrel{P}{\longrightarrow} 1
$$

for $d=d_{n} \rightarrow \infty, d_{n} / n \rightarrow 0$. Fix $n \geq 1,1 / 2<t<2$ and let $T_{k}=I\left\{X_{k} \geq t n / d\right\}, 1 \leq k \leq n$. Then by Lemma 2.2 and (1.11) we get

$$
\left|\mathrm{E} T_{1} T_{k}-\mathrm{E} T_{1} \mathrm{E} T_{k}\right| \leq \psi(k-1) \mathrm{E} T_{1} \mathrm{E} T_{k} \leq C_{9} \exp (-\lambda k)(d / n)^{2}
$$

and thus setting $\bar{T}_{k}=T_{k}-E T_{k}$ we conclude that

$$
\begin{aligned}
\mathrm{E}\left(\sum_{k=1}^{n} \bar{T}_{k}\right)^{2} & =n \mathrm{E} \bar{T}_{1}^{2}+2 \sum_{k=2}^{n}(n-k+1) \mathrm{E} \bar{T}_{1} \bar{T}_{k} \\
& \leq n\left(\mathrm{E} \bar{T}_{1}^{2}+2 \sum_{k=2}^{n}\left|\mathrm{E} \bar{T}_{1} \bar{T}_{k}\right|\right) \\
& \leq n\left(\mathrm{E} T_{1}^{2}+C_{10}(d / n)^{2} \sum_{k=2}^{n} \exp (-\lambda k)\right) \\
& \leq n\left(\mathrm{E} T_{1}+C_{11}(d / n)^{2}\right) \\
& \leq C_{12} d .
\end{aligned}
$$

Hence Markov's inequality and $d=d_{n} \rightarrow \infty$ imply for any $\varepsilon>0$

$$
P\left\{\sum_{k=1}^{n} \bar{T}_{k} \geq \varepsilon d\right\} \longrightarrow 0,
$$


and since $\mathrm{E} T_{k}=\mathrm{E} T_{1} \sim d /(n t)$ by (1.11), it follows that

$$
\#\left\{k \leq n: X_{k} \geq t n / d\right\}=\sum_{k=1}^{n} I\left\{X_{k} \geq t n / d\right\} \sim d / t \quad \text { in probability as } n \rightarrow \infty .
$$

Thus for fixed $t>1$ and $n$ large, with probability tending to 1 the number of $X_{k}$ 's, $1 \leq k \leq n$ exceeding $t n / d$ is smaller than $d$, and thus $\eta_{d, n} \leq t n / d$. Similarly, for $t<1$ and $n$ large, with probability tending to 1 we have $\eta_{d, n} \geq t n / d$, and thus (3.15) is proved.

Proof of Remark 1.1. Let $\left(X_{n}\right)$ be a sequence satisfying the assumptions of Theorem 1.2, and put $X_{n}^{\prime}=X_{n}+4^{-n}$. Letting $\eta_{d, n}^{\prime}$ denote the $d$-th largest of $X_{1}^{\prime}, \ldots, X_{n}^{\prime}$ and $S_{n}^{(r)}$ and $S_{n}^{\prime(r)}$ denote the sums $\sum_{k=1}^{n} X_{k}, \sum_{k=1}^{n} X_{k}^{\prime}$ after removing their $r$ largest terms, it is easily seen that

$$
\left|S_{n}^{(r)}-S_{n}^{\prime(r)}\right| \leq 2 \quad \text { for any } r \geq 1
$$

and

$$
n\left|m\left(\eta_{d, n}\right)-m\left(\eta_{d, n}^{\prime}\right)\right|=O_{P}(1)
$$

Clearly, relation (1.11) will fail for the perturbed sequence $\left(X_{n}^{\prime}\right)$, but as inspection shows, all the lemmas in the proof of Theorem 1.2 and the subsequent arguments remain valid, so conclusion (1.13) of the theorem remains valid if we replace $X_{i}$ by $X_{i}^{\prime}$ and $\eta_{d, n}$ by $\eta_{d, n}^{\prime}$. Since the $X_{n}$ are integer valued, with probability one all the $X_{j}^{\prime}, j=1,2, \ldots$ are different, and thus the sum of the $X_{j}$ 's, $1 \leq j \leq n$ not exceeding $\eta_{d, n}^{\prime}$ equals $S_{n}^{\prime(d-1)}$. Thus we have

$$
\frac{S_{n}^{(d-1)}-n m\left(\eta_{d, n}^{\prime}\right)}{n / \sqrt{d}} \stackrel{d}{\longrightarrow} N\left(0, c_{0}\right) .
$$

In view of (3.16) and (3.17), we can drop the primes in (3.18) and since $S_{n}^{(d-1)}-S_{n}^{(d)}=\eta_{d, n}=$ $O_{P}(n / d)$ by $(3.15)$, the conclusion of Theorem 1.1 follows.

Acknowledgement. We would like to thank the referee for her/his remarks leading to a substantial improvement of the paper.

\section{REFERENCES}

[1] Arov, D.Z. and Bobrov, A.A.: The extreme terms of a sample and their role in the sum of independent variables. Theor. Probability Appl. 5 (1960), 377-396.

[2] Bickel, P. J. and Wichura, M. J.: Convergence criteria for multiparameter stochastic processes and some applications. Ann. Math. Statist. 42 (1971), 1656-1670.

[3] Billingsley, P.: Ergodic theory and information. Wiley, 1965.

[4] Billingsley, P.: Convergence of Probability Measures. Wiley, New York 1968.

[5] Bradley, R.: Introduction to strong mixing conditions, Vol I. Kendrick Press, 2007.

[6] Csörgő, S., Horváth, L. and Mason, D.: What portion of the sample makes a partial sum asymptotically stable or normal ? Z. Wahrschein. verw. Gebiete 72 (1986), 1-16.

[7] Csörgö, S. and Simons, G.: A strong law of large numbers for trimmed sums, with applications to generalized St. Petersburg games. Statist. Prob. Letters 26 (1996), 65-73.

[8] Darling, D.: The influence of the maximum term in the addition of independent random variables. Trans. Amer. Math. Soc. 73 (1952), 95-107. 
[9] Diamond, H. and Vaaler, J.: Estimates for partial sums of continued fraction partial quotients. Pacific J. Math. 122 (1986), 73-82.

[10] Doeblin, W.: Remarques sur la théorie métrique des fractions continues. Comp. Math. 7 (1940), 353-371.

[11] Galambos, J.: The distribution of the largest coefficient in continued fraction expansions. Quart. J. Math. Oxford Ser. 23 (1972), 147-151.

[12] Galambos, J.: An iterated logarithm type theorem for the largest coefficient in continued fractions. Acta Arith. $25(1973 / 74), 359-364$.

[13] Gordin, M.I. and Reznik, M.H.: The law of the iterated logarithm for the denominators of continued fractions. Vestnik Leningrad Univ. 25 (1970), 28-33. (In Russian)

[14] Heinrich, L.: Rates of convergence in stable limit theorems for sums of exponentially $\psi$ - mixing random variables with an application to metric theory of continued fractions. Math. Nachr. 131 (1987), 149-165.

[15] Ibragimov, I.A.: A theorem from the metric theory of continued fractions. Vestnik Leningrad Univ. 1 (1960), 13-24. (In Russian)

[16] Iosifescu, M.: A Poisson law for $\psi$-mixing sequences establishing the truth of a Doeblin's statement. Rev. Roumaine Math. Pures Appl. 22 (1977), 1441-1447.

[17] Iosifescu, M.: A survey of the metric theory of continued fractions, fifty years after Doeblins 1940 paper. In: Probability Theory and Mathematical Statistics, B. Grigelionis et al. (Eds.) Vol. I, pp. 550-572. Vilnius, Mokslas (1990)

[18] Khinchin, A. J.: Metrische Kettenbruchprobleme. Compositio Math. 1 (1935), 361-382.

[19] Kusmin, R.: Sur un problème de Gauss. Atti Congr. Intern. Bologne 1928. 6, 83-89.

[20] Leadbetter, M. and Rootzén, H.: Extremal theory for stochastic processes. Ann. Probab. 16 (1988), 431-478.

[21] Lévy, P.: Sur les lois de probabilité dont dépendent les quotients complets et incomplets d'une fraction continue. Bull. Sci. Math. France 57 (1929), 178-194.

[22] Lévy, P.: Fractions continues aléatoires. Rend. Circ. Mat. Palermo 1 (1952), 170-208.

[23] Philipp, W.: Some metrical theorems in number theory II. Duke Math. J. 37 (1970), 447-458.

[24] Philipp, W.: A conjecture of Erdős on continued fractions. Acta Arith. 28 (1975/76), 379-386.

[25] Philipp, W.: Limit theorems for partial quotients of continued fractions. Monatsh. Math. 105 (1988), $195-206$.

[26] Philipp, W. and Stackelberg. O.: Zwei Grenzwertssätze für Kettenbrüche. Math. Ann. 181 (1969), $152-156$.

[27] Samur, J.: On some limit theorems for continued fractions. Trans. Amer. Math. Soc. 316 (1989), 53-79.

[28] Samur, J.: Some remarks on a probability limit theorem for continued fractions. Trans. Amer. Math. Soc. 348 (1996), 1411-1428.

[29] Stackelberg, O.P.: On the law of the iterated logarithm for continued fractions. Duke Math. J. 33 (1966), $801-819$.

[30] Szewczak, Z.S.: On limit theorems for continued fractions. J. Theor. Probab. 22 (2009), 239-255.

[31] Utev, S. A.: On the central limit theorem for $\varphi$-mixing arrays of random variables. Theory Probab. Appl. 35 (1990), 131-139.

[32] Vardi, I.: The St. Petersburg game and continued fractions. C.R. Acad. Sci. Paris Ser. I. Math. 324 (1997), 913-918. 